\title{
Therapeutic potential of hyaluronic acid/chitosan nanoparticles for the delivery of curcuminoid in knee osteoarthritis and an in vitro evaluation in chondrocytes
}

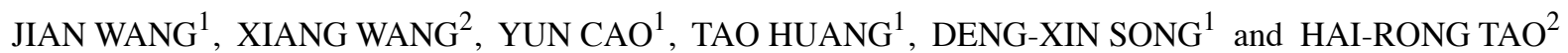 \\ ${ }^{1}$ Department of Orthopedics, Baoshan Branch of Shanghai First People's Hospital, Shanghai 200940; \\ ${ }^{2}$ Department of Orthopaedics, Shanghai Ninth People's Hospital, Shanghai Jiao Tong University \\ School of Medicine, Shanghai 201999, P.R. China
}

Received March 28, 2018; Accepted August 6, 2018

DOI: $10.3892 /$ ijmm.2018.3817

\begin{abstract}
Knee osteoarthritis (OA) is the main cause of leg pain in middle-aged and elderly individuals. Hyaluronic acid (HA), as well as curcuminoid, has been used in the treatment of knee OA. In the present study, HA/chitosan nanoparticles (CNPs) were prepared for the delivery of curcuminoid, in order to investigate whether HA and curcuminoid can act synergistically as a better treatment option. The knee OA model was established by the Hulth method, and a knee OA chondrocyte model was constructed by the co-induction of interleukin- $1 \beta$ and tumor necrosis factor (TNF)- $\alpha$. The drug loading capacity of HA/CNP for the delivery of curcuminoid was measured by an ultraviolet assay, and the cytotoxicity to chondrocytes was measured by an MTT assay. Collagen II was detected by immunofluorescence, and the expression levels of nuclear factor (NF)- $\mathrm{kB}$ and inflammation-related genes in cartilage tissue and chondrocytes were detected. Chondrocyte proliferation was determined by an EdU assay, and chondrocyte apoptosis was determined by flow cytometry. The Mankin pathological score of the Outerbridge classification was obtained. The results demonstrated that the optimum drug loading capacity of HA/CNP for the delivery of curcuminoid was $38.44 \%$, with a good sustained release function. HA/CNP treatment resulted in inhibition of the NF- $\mathrm{\kappa B}$ pathway, as well as the expression of matrix metalloproteinase (MMP)-1 and MMP-13, but it increased collagen II expression. HA/CNP for the delivery of curcuminoid significantly decreased the Outerbridge classification and Mankin pathological scores to
\end{abstract}

Correspondence to: Dr Hai-Rong Tao, Department of Orthopaedics, Shanghai Ninth People's Hospital, Shanghai Jiao Tong University School of Medicine, 280 Mohe Road, Shanghai 201999, P.R. China

E-mail: taohair@163.com

Key words: knee osteoarthritis, hyaluronic acid/chitosan nanoparticles for the delivery of curcuminoid, inflammation, nuclear factor- $\kappa \mathrm{B}$, matrix metalloproteinase-1, matrix metalloproteinase-13 close to normal until the 4th week. Furthermore, it was also observed that all the effects of HA/CNP on the delivery of curcuminoid were more prominent compared with the effects of HA or curcuminoid treatment individually. Taken together, these findings demonstrated that HA/CNP for the delivery of curcuminoid may suppress inflammation and chondrocyte apoptosis in knee OA via repression of the NF-kB pathway.

\section{Introduction}

Osteoarthritis (OA) is a common type of degenerative arthritis that is characterized by subchondral bone damage, synovitis and articular cartilage degradation (1). Due to joint pain, inflammation, movement difficulty and crepitus, knee OA poses a major burden on patient daily life, and even causes disability (2). The World Health Organization has reported that $>10 \%$ of the population aged $<60$ years suffer from $\mathrm{OA}$ worldwide (3). Nuclear factor (NF)- $\mathrm{B}$ was reported to enhance the synthesis of nitric oxide and other catabolic factors that are drivers of OA chondrocyte apoptosis and cartilage inflammation (4). A previous study demonstrated that in vitro injection or oral administration of hyaluronic acid (HA) may be a viable treatment option for patients with knee OA (5). Furthermore, it has also been demonstrated that curcuminoid inhibited the progression of $\mathrm{OA}$ and effectively alleviated pain symptoms (6). Therefore, the aim of the present study was to examine whether HA and curcuminoid can act synergistically to provide the best therapeutic efficacy in knee OA.

$\mathrm{HA}$ is a mucopolysaccharide biopolymer forming from cyclical units of disaccharides (7). Intra-articular HA is often applied in knee OA treatment to relieve pain and reduce knee inflammation (8). Moreover, evidence from a recent study suggests that curcuminoid inhibited chondrocyte apoptosis and the inflammatory signaling pathway in OA (9). However, the use of curcuminoid is limited in therapeutics due to its low aqueous solubility, unstable chemical characteristics, and poor bioavailability following oral administration. However, the low bioavailability of curcuminoid may be improved by nanoparticles (10). In addition, chitosan with high molecular weight may improve colloidal stability during the preparation of curcuminoid nanoplexes and does not substantially affect the 
dissolution capacity for other materials (11). Furthermore, HA interacts with intercellular adhesion molecule-1 to decrease Akt expression in a lipopolysaccharide-stimulated macrophage cell line, and may also inhibit NF- $\kappa \mathrm{B}$ pathway activation (12). Curcumin significantly inhibited matrix metalloproteinase (MMP)-13 mRNA and suppressed NF- $\mathrm{KB}$ pathway activation by repressing $\mathrm{I}-\kappa \mathrm{B} \alpha$ phosphorylation and degradation, as well as P65 nuclear translocation (13). In addition, inhibition of the $\mathrm{NF}-\kappa \mathrm{B}$ pathway was reported to attenuate inflammation (14). Thus, it was hypothesized that HA and curcuminoid may be better dissolved with the help of chitosan and nanoparticles to form HA/chitosan nanoparticles (CNP) for the delivery of curcuminoid, and that HA/CNP for the delivery of curcuminoid can treat knee OA by targeting the $N F-\kappa B$ pathway. The aim of the present study was to determine whether the use of intra-articular HA/CNP for the delivery of curcuminoid has a better efficacy compared with HA or curcuminoid treatment individually, in order to investigate new approaches to the treatment of knee OA.

\section{Materials and methods}

Ethics statements. The breeding and sacrifice of the experimental animals, as well as tissue retrieval, were all for medical research and performed in strict accordance with the recommendations in the Guide for the Care and Use of Laboratory Animals of the National Institutes of Health. The procedures were approved by the Animal Committee of Shanghai Ninth People's Hospital, Shanghai Jiao Tong University School of Medicine.

Study subjects. A total of 70 specific-pathogen-free (SPF) healthy male Sprague Dawley (SD) rats (age, 8 weeks; weight range, 180-220 g) were provided by SLAC Laboratory Animal Co. Ltd. (Shanghai, China). The rats were housed in an environment of $68 \%$ humidity at $25^{\circ} \mathrm{C}$ with $12 \mathrm{~h}$ of light every day, with free access to food and running water supplied by the feeders.

Establishment of animal model. The Hulth method was applied to establish a knee OA model in SD rats (15). After a 12-h fasting period, the SD rats were fully anesthetized by intraperitoneal injection of $0.3 \%$ pentobarbital sodium (Sigma-Aldrich; Merck KGaA, Darmstadt, Germany ) at $10 \mathrm{ml} / \mathrm{kg}$ and were then fixed in the supine position. The patellae were pushed outward until dislocated. When the knees were at maximum bending angle, the articular cavity was exposed, and the right knee anterior cruciate ligaments (ACLs), medial collateral ligaments (MCLs) and medial menisci were cut off without any damage to the joint cartilage surface. After the knee was determined to be positive by the drawer test, the wounds were fully stanched and sutured layer by layer, while treated with drops of penicillin solution (Sigma-Aldrich; Merck KGaA). After the operation, the rats were treated by intramuscular injection of 40,000 units of penicillin daily for 4 days. On the fifth day, the rats were forced to run for $30 \mathrm{~min}$ per day. The sham group consisted of SD rats which had undergone articular cavity surgery without any damage to the ACL, MCL or medial menisci. The subsequent treatments were the same as those in the rats in the OA group. After being forced to run every day for 4 weeks, the walking status of the rats was observed, and their joints were examined by safranin $\mathrm{O}$-fast green staining. There were $5 \mathrm{SD}$ rats in the sham group and 5 SD rats in the OA group; a total of 60 rats (20 rats per group) were used for the subsequent experiments.

Chondrocyte isolation and culture. Ten SD rats were euthanized using $100 \% \mathrm{CO}_{2}$, were immersed in $75 \%$ ethanol for $10 \mathrm{~min}$, and were then transferred to a superclean bench. The tibial cartilages of the knee joints were removed using a surgical knife and washed with phosphate-buffered saline (PBS) 3 times. The cartilage was cut into pieces, collected in a 50-ml centrifuge tube, and treated with $2 \mathrm{ml} 0.25 \%$ trypsin (Sigma-Aldrich; Merck KGaA) for $60 \mathrm{~min}$. The trypsin was then removed, and the cartilages were rinsed twice with PBS and then treated with $0.2 \%$ type II collagenase (Sigma-Aldrich; Merck $\mathrm{KGaA}$ ) for $4 \mathrm{~h}$. After removal of the collagenase, turbid liquid was poured into a 120-pore mesh screen (Solarbio Science and Technology Ltd., Co., Beijing, China) and the filtered liquid was collected and centrifuged at $100 \mathrm{x} \mathrm{g}$ for $10 \mathrm{~min}$. Subsequently, the filtered liquid was washed with PBS twice and incubated in Dulbecco's Modified Eagle's Medium (DMEM) (Gibco; Thermo Fisher Scientific, Inc., Waltham, MA, USA ) containing 10\% fetal bovine serum (FBS) (Gibco; Thermo Fisher Scientific Inc.) to resuspend cells. The cell resuspension was evenly inoculated in a $35-\mathrm{mm}$ culture dish and cultured in a $5 \% \mathrm{CO}_{2}$ incubator at $37^{\circ} \mathrm{C}$. Subsequently, the growth of chondrocytes was observed under an inverted microscope (Promega Corporation, Madison, WI, USA). When confluence reached $90 \%$, separated primary chondrocytes were passaged.

Immunofluorescence method. Chondrocytes were digested, resuspended, replated in a 6-well plate with an adhesive coverglass inside at density of $1 \times 10^{6}$ cells/well, and then cultured in a $5 \% \mathrm{CO}_{2}$ incubator at $37^{\circ} \mathrm{C}$. The coverglass was removed when the cells reached $90 \%$ confluence. The cells were washed with PBS, fixed with $1 \mathrm{ml} \mathrm{4 \%}$ paraformaldehyde for $15 \mathrm{~min}$, rinsed twice with PBS, and permeabilized with $1 \mathrm{ml} 0.2 \%$ Triton-PBS for $30 \mathrm{~min}$. The cells were then rinsed twice with

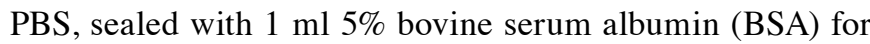
$20 \mathrm{~min}$, and incubated in $1 \mathrm{ml}$ rabbit anti-rat collagen II (1:100, ab34712, Abcam, Cambridge, MA, USA) at $4^{\circ} \mathrm{C}$ overnight. The cells were rinsed with PBS and then incubated with secondary goat anti-rabbit antibody immunoglobulin $\mathrm{G}(\mathrm{IgG} ; 1: 1,000$, ab150077, Abcam) at room temperature for $4 \mathrm{~h}$ in the dark. Cells were rinsed twice with PBS, stained with $100 \mu$ l Hoechst 33258 staining solution at room temperature for $5 \mathrm{~min}$, and washed twice with PBS. Then, the cells were fast-rinsed with 95\% ethanol and dried. An appropriate amount of fluorescence quenching solution was dropped onto the glass slide, and then the coverslip was applied. Subsequent fluorescence was observed using an inverted fluorescence phase-contrast microscope (EclipseTi-S, Nikon, Tokyo, Japan).

Ionic cross-linking method. Chitosan (9 mg, 10-40 kD, Jinke Company, Zhejiang, China) was precisely weighed, collected, dissolved in $3.6 \mathrm{ml}$ distilled water, and added to $5 \mathrm{ml}$ curcuminoid solution $(1 \mathrm{mg} / \mathrm{ml}$ in absolute ethanol, Geleixiya Company, Chengdu, China) for $10 \mathrm{~min}$. This reaction mixture was added to $1 \mathrm{ml}$ triphenyl phosphate $(2 \mathrm{mg} / \mathrm{ml}$ in water) 
(Sangon Biotech Company, Shanghai, China) and incubated at room temperature for $30 \mathrm{~min}$. The suspension was then centrifuged at $23,649 \times \mathrm{g}$ at $4^{\circ} \mathrm{C}$ for $40 \mathrm{~min}$. Sediments were collected, washed and treated with lyophilization to obtain a powder. A total of $30 \mathrm{ml}$ distilled water was added into a 50-ml centrifuge tube. Then, $90 \mathrm{mg}$ HA (Sangon Biotech Company), $180 \mathrm{mg}$ endocrine-disrupting chemical (Sangon Biotech Company) and $195 \mathrm{mg}$ N-hydroxysuccinimide (Sangon Biotech Company) were added in that order, and the solution was adjusted to $\mathrm{pH}$ 7.5. The tube was incubated in a water bath at $37^{\circ} \mathrm{C}$ for $3 \mathrm{~h}$. Then, $15 \mathrm{mg}$ of the aforementioned lyophilized powder was added, and the mixture was incubated for $24 \mathrm{~h}$ at $300 \mathrm{x} \mathrm{g}$ to obtain a nanosuspension. The nanosuspension was centrifuged at $23,469 \times \mathrm{g}$ at $4^{\circ} \mathrm{C}$ for $60 \mathrm{~min}$, and sediments were obtained. Finally, HA/CNP for the delivery of curcuminoid was obtained after the sediments were washed and treated via lyophilization. The molecular weight of the resulting compound was 500-1,000 kD.

Morphological observation of $H A / C N P$ for the delivery of curcuminoid. The appropriate amount of HA/CNP for the delivery of curcuminoid powder was diluted in distilled water, dropped on a copper grid-covered carbon film, and incubated with $2 \%$ phosphotungstic acid for $10 \mathrm{sec}$. The reaction products were dried and observed under a transmission electron microscope (TEM) (Hitachi Ltd., Tokyo, Japan).

Ultraviolet (UV) assay. Curcuminoid solutions were prepared in absolute ethanol at concentrations of 1, 2, 3, 4, 5 and $6 \mathrm{~g} / \mathrm{ml}$. The optical densities (ODs) of each solution were measured at $430 \mathrm{~nm}$ wavelength with a blank control of absolute ethanol. From these data, a standard curve was calculated. Nanoparticles of freeze-dried HA/CNPs were destroyed by $10 \%$ hydrochloric acid, and HA/CNPs were then diluted with absolute ethanol to $10 \mathrm{ml}$. ODs were measured at $430 \mathrm{~nm}$ to calculate the drug loading capacity.

Bag filter method. HA/CNPs were collected and then suspended in $3 \mathrm{ml}$ PBS containing 1\% sodium dodecyl sulfate (SDS) $(0.2 \mathrm{~mol} / 1, \mathrm{pH} 6.6)$ to adjust the drug concentration to $0.4 \mathrm{mg} / \mathrm{ml}$. The final solution was put in a dialysis bag (Sinopharm Chemical Reagent Co., Ltd., Shanghai, China) (Mr2000). The dialysis bag was then placed in a beaker with $30 \mathrm{ml} \mathrm{PBS}$ and incubated in a water bath at $37^{\circ} \mathrm{C}$, while shaking at $60 \mathrm{x} \mathrm{g}$. A total of $1 \mathrm{ml}$ of the solution was collected after $1,2,4,8,12,18,24,36,48,60$ and $72 \mathrm{~h}$ (isothermal medium was appended), and the OD values at $430 \mathrm{~nm}$ were measured. These values were drawn into a standard curve and used to calculate related concentrations and total release rates of the samples in two groups at each time point.

3-(4,5-dimethylthiazol-2-yl)-2,5-diphenyltetrazolium bromide (MTT) assay. SD rat chondrocytes $\left(3.0 \times 10^{4}\right.$ cells $/ \mathrm{ml}$ diluted in $10 \%$ FBS/DMEM) were inoculated in a 96-well culture plate. Each group had three duplicates with $100 \mu \mathrm{l}$ in each well. The cells were cultured in $5 \% \mathrm{CO}_{2}$ at $37^{\circ} \mathrm{C}$ for $24 \mathrm{~h}$. A total of $100 \mu \mathrm{l}$ of media containing different concentrations of curcuminoid $(4,8,16,32$ and $64 \mu \mathrm{g} / \mathrm{ml})$ and HA/CNPs $(8,16,32,64$ and $128 \mu \mathrm{g} / \mathrm{ml}$ ) was added to each well, and the cells were cultured for 24 and $48 \mathrm{~h}$. Then, $20 \mu \mathrm{l} \mathrm{MTT}(5 \mathrm{mg} / \mathrm{ml})$ was added to
$80 \mu 1$ serum-free medium, and the mixture was added to the cells at different time points. The cells were incubated in a 5\% $\mathrm{CO}_{2}$ incubator at $37^{\circ} \mathrm{C}$ for $4 \mathrm{~h}$, treated with $150 \mu \mathrm{l}$ dimethylsulphoxide and rocked on a shaking table for $10 \mathrm{~min}$ until the crystals were fully dissolved. The OD values of each well were measured at $570 \mathrm{~nm}$ by a microplate reader for the calculation of chondrocyte $\mathrm{IC}_{50}$.

Cell model construction. After the third passage, chondrocytes were plated into a 6 -well plate at a density of $1 \times 10^{6}$ cells/well, and then the medium was replaced with DMEM complete medium containing $10 \mathrm{ng} / \mathrm{ml}$ interleukin (IL)-1 $\beta$ (Sigma-Aldrich; Merck KGaA) and $50 \mathrm{ng} / \mathrm{ml}$ tumor necrosis factor (TNF)- $\alpha$ (Sigma-Aldrich; Merck KGaA). After 12 h, drugs were added (curcuminoid as the benchmark) as follows: The negative control (NC) group was treated with fresh culture medium, the HA group was treated with complete medium containing $10 \mu \mathrm{g} / \mathrm{ml} \mathrm{HA}$, the curcuminoid group was treated with complete medium containing $10 \mu \mathrm{g} / \mathrm{ml}$ curcuminoid, and the HA/CNP group was treated with complete medium containing $30 \mu \mathrm{g} / \mathrm{ml} \mathrm{HA} / \mathrm{CNP}$. The chondrocytes were further cultured for $48 \mathrm{~h}$ for subsequent experiments.

5-Ethynyl-2'-deoxyuridine (EdU) assay. SD rat chondrocytes in the logarithmic growth phase were collected and plated in a 96-well plate at a density of 3,000 cells/well. The NC, $\mathrm{HA}$, curcuminoid and HA/CNP groups were analyzed in three experiments of technical duplicates. Chondrocytes were cultured in a thermostatic incubator for $68 \mathrm{~h}$ and treated with $20 \mu \mathrm{mol} / \mathrm{l}$ EdU solution for $4 \mathrm{~h}$. The chondrocytes were fixed with $4 \%$ paraformaldehyde for $15 \mathrm{~min}$, and $0.5 \%$ Triton X-100 was added to permeabilize the membrane. The cells were then incubated with Click-iT ${ }^{\mathrm{TM}}$ in the dark for $30 \mathrm{~min}$. Finally, the nuclei were stained with Hoechst 33342, photographed and counted under a fluorescence microscope. Five fields from each well were randomly selected under the microscope. Cells that stained positive for EdU were proliferating. The cell proliferation rate was calculated as the number of EdU-positive cells/the number of total cells.

Flow cytometry. After 48 h of drug treatment, the cells were collected in flow tubes and centrifuged at 1,000 x $\mathrm{g}$ for $5 \mathrm{~min}$. Then, the cells were washed three times with cold PBS and centrifuged. According to the instructions of Annexin V-FITC Cell Apoptosis assay kit (Sigma-Aldrich; Merck KGaA), cells in each tube were added to $150 \mu \mathrm{l}$ binding buffer and $5 \mu \mathrm{l}$ Annexin V-FITC, shaken to completely mix, and incubated at room temperature in the dark for $15 \mathrm{~min}$, after which cells in each tube were combined with $100 \mu \mathrm{l}$ binding buffer and $5 \mu \mathrm{l}$ propidium iodide (PI) dye (Sigma-Aldrich; Merck KGaA), and shaken to completely mix. Flow cytometers (FACSCanto II, BD Biosciences, Franklin Lakes, NJ, USA) were used to detect cell apoptosis.

Western blot analysis. Cartilage tissues and chondrocytes were dissociated in RIPA lysis buffer (Beyotime Institute of Biotechnology, Shanghai, China), and centrifuged at $14,000 \times \mathrm{g}$ at $4^{\circ} \mathrm{C}$. The supernatant was collected to obtain total protein, and preserved at $-80^{\circ} \mathrm{C}$. Nuclear and cytoplasmic proteins from chondrocytes were obtained using 
the Cell nuclear protein and Cytoplasmic Protein Extraction Kits according to the manufacturer's instructions (Beyotime Institute of Biotechnology). Then, the proteins were stored at $-80^{\circ} \mathrm{C}$. A bicinchoninic acid assay (Pierce; Thermo Fisher Scientific, Inc.) was used to assess the protein concentration of the protein extraction solution. Proteins were separated by electrophoresis on a $4 \%$ spacer gel and a $10 \%$ a spacer gel, and transferred onto a membrane. The membrane was blocked with $0.5 \%$ BSA and incubated with the following primary antibodies overnight: rabbit anti-rat collagen II (1:1,000; ab34712; Abcam), rabbit anti-rat MMP-13 (1:1,000; ab39012; Abcam), rabbit anti-rat $\mathrm{I}-\kappa \mathrm{B} \alpha(1: 1,000 ; \mathrm{ab} 7217$; Abcam), rabbit anti-rat

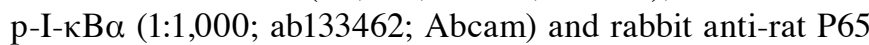
(1:1,000; ab16502; Abcam). The membrane was washed and incubated with horseradish peroxidase-labeled goat anti-rabbit immunoglobulin G (1:1,000; ab205718; Abcam) at room temperature for $2 \mathrm{~h}$, and then developed with enhanced chemiluminescence (Invitrogen; Thermo Fisher Scientific, Inc.). The images were obtained using the Bio-Rad microscopic imaging system (Bio-Rad Laboratories, Inc., Hercules, CA, USA) and analyzed by ImageJ Software. The experiment was performed in triplicate.

Reverse transcription-quantitative polymerase chain reaction $(R T-q P C R)$. Cartilage tissues and chondrocytes were ground using a mortar in liquid nitrogen, and RNAs were extracted with TRIzol (Invitrogen; Thermo Fisher Scientific, Inc.). RNA concentration was measured using a spectrophotometer. RNA was reverse-transcribed into cDNA through oligodT assay. RT-qPCR was performed with a SYBR Green assay. The reaction system was as follows: $1.0 \mu \mathrm{l} \mathrm{cDNA}, 10 \mu \mathrm{l} 2 \mathrm{X}$ SYBR Green Mix, $0.5 \mu \mathrm{l}$ forward primer $(10 \mu \mathrm{M}), 0.5 \mu \mathrm{l}$ reverse primer $(10 \mu \mathrm{M})$ and RNase-free water to a total of $20 \mu \mathrm{l}$. The reaction conditions were as follows: $95^{\circ} \mathrm{C}$ for $3 \mathrm{~min}, 40$ cycles of $95^{\circ} \mathrm{C}$ for $15 \mathrm{sec}, 60^{\circ} \mathrm{C}$ for $30 \mathrm{sec}$ and $72^{\circ} \mathrm{C}$ for $20 \mathrm{sec}$, and then $72^{\circ} \mathrm{C}$ for $5 \mathrm{~min}$. Primers were synthesized by the Beijing Genomics Institute (Beijing, China) (Table I). The solubility curve was used to evaluate the reliability of the PCR results, while a quantitative method was applied for calculation. The $2^{-\mathrm{ACq}}$ value represented the relative expression of each target gene (16). Each experiment was performed in triplicate to obtain the mean value.

Collection of knee articular tissues. HA and HA/CNP for the delivery of curcuminoid were prepared at a $10 \mathrm{mg} / \mathrm{ml}$ solution with normal saline. At 4 weeks after knee OA model establishment, the knee articular cavity was injected with either $0.1 \mathrm{ml}$ drugs or normal saline every week for 4 weeks. A total of $5 \mathrm{SD}$ rats were euthanized by cervical dislocation each week, and their knee-joint tissues were collected, photographed and quickly stored in liquid nitrogen.

Hematoxylin and eosin $(H \& E)$ staining. Rat knee joints were fixed with $10 \%$ formaldehyde solution for $24 \mathrm{~h}$, embedded in paraffin, and cut into sections. The sections were dewaxed with xylene, dehydrated using an ethanol gradient (100\% I, 100\% II, 95, 85 and 75\%; 3 min for each concentration), and soaked in hematoxylin dye liquor for $5 \mathrm{~min}$. The sections were then washed with running water for $1 \mathrm{~min}$ to remove floating color, dipped in $1 \% \mathrm{NS}$ ethanol for $30 \mathrm{sec}$, and washed with running water for $1 \mathrm{~min}$. Next, the sections were immersed in $0.5 \%$
Table I. Primers for RT-qPCR

\begin{tabular}{ll}
\hline Gene & \multicolumn{1}{c}{ Sequence } \\
\hline MMP-1 & F: CGGCAGAATGTGGAA \\
& R: AACGAGGATTGTTGTGAGTA \\
MMP-13 & F: GCCATTACTCACAACAATCCTCG \\
& R: AACACAATATCACCTTCCTCCTCAA \\
Collagen II & F: TCCGCTGGTCTGATGGACAC \\
& R: CCAGATCATCACTACGCAGTCCTC \\
GAPDH & F: CCCCAATGTATCCGTTGTG \\
& R: CTCAGTGTAGCCCAGGATGC \\
\hline
\end{tabular}

RT-qPCR, reverse transcription quantitative polymerase chain reaction; $\mathrm{F}$, forward; $\mathrm{R}$, reverse; MMP, matrix metalloproteinase; GAPDH, glyceraldehyde-3-phosphate dehydrogenase.

eosin dye liquor for $3 \mathrm{~min}$, rinsed with running water for $1 \mathrm{~min}$, dehydrated using an ethanol gradient $(75,85,95,100 \%$ II and $100 \% \mathrm{I} ; 3 \mathrm{~min}$ for each concentration), cleared by xylene, and sealed by neutral gum.

Toluidine blue staining. Rat knee joints were fixed with $10 \%$ formaldehyde solution for $24 \mathrm{~h}$, embedded in paraffin, and cut into sections. The sections were dewaxed by xylene, dehydrated using an ethanol gradient (100\% I, 100\% II, 95, 85 and 75\%; 3 min for each concentration), and soaked in $2 \%$ toluidine blue dye liquor for $10 \mathrm{~min}$. After that, the sections were rinsed with running water for $1 \mathrm{~min}$ to remove floating color, dehydrated using an ethanol gradient $(75,85,95,100 \%$ II and $100 \%$ I; $3 \mathrm{~min}$ for each concentration), cleared by xylene, and sealed with neutral gum.

Safranin $O$-fast green staining. The experiment was performed according to the instructions of the Safranin O-fast green reagent manufacturer (Newcomer Supply, Middleton, WI, USA). Rat knee joints were fixed with $10 \%$ formaldehyde solution for $24 \mathrm{~h}$, embedded in paraffin, and cut into sections. The sections were dewaxed by xylene, dehydrated using an ethanol gradient $(100 \%$ I, 100\% II, 95, 85 and 75\%; 3 min for each concentration), and soaked in safranin O-fast green dye liquor for $10 \mathrm{~min}$. After that, the sections were rinsed with running water for $1 \mathrm{~min}$ to remove floating color, dehydrated using an ethanol gradient $(75,85,95$, $100 \%$ II and $100 \%$ I; 3 min for each concentration), cleared by xylene, and sealed with neutral gum.

Mankin pathological score of Outerbridge classification. Three orthopedists were invited by Shanghai Ninth People's Hospital, Shanghai Jiao Tong University School of Medicine to individually assess SD rat knee joints according to Tables II and III. From this assessment, the Mankin pathological score of Outerbridge classification was obtained.

Statistical analysis. SPSS21.0 (IBM Corp. Armonk, NY, USA) was used to analyze data and calculate the mean value and standard deviation. All experiments were repeated at least 3 times. Differences between two groups were compared by 
Table II. Outerbridge classification of rat knee joints.

$\begin{array}{ll}\text { Outerbridge class Pathological characteristics } & \text { Pate }\end{array}$

$\begin{array}{ll}0 & \text { Joint cartilage is light white, translucent, glossy, without cracks or erosions. } \\ \text { I } & \text { Joint cartilage is yellowish, with flat surface, and mostly without cracks or erosions. } \\ \text { II } & \text { Joint cartilage has visible cracks and erosions, and is thinner. } \\ \text { III } & \text { Joint cartilage is of a darker color and bears an obvious fracture with the lower layer. }\end{array}$

Table III. Mankin pathological score of rat knee joints.

\begin{tabular}{lcllll}
\hline Cartilage structure & Score & & Cellularity & Score & \multicolumn{2}{c}{ Tideline completeness } \\
\hline Normal & 0 & Normal & 0 & Fully complete & Score \\
Cracks only on the surface & 1 & Diffuse hypercellularity & 1 & Multiple tideline & 1 \\
Cracks in transition layer & 2 & Local cell clustering & 2 & Tideline deep into cartilage blood vessel & 2 \\
Cracks in emission layer & 3 & Hypocellularity & 3 & \\
Cracks in calcified cartilage zone & 4 & & & \\
Cartilaginous sclera disappeared & 5 & & & \\
\hline
\end{tabular}

the t-test. Multiple groups were compared by one-way analysis of variance (ANOVA). $\mathrm{P}<0.05$ was considered to indicate statistically significant differences.

\section{Results}

HA/CNP for the delivery of curcuminoid promotes chondrocyte proliferation and has sustained release action. The optimum drug loading capacity of HA/CNP for the delivery of curcuminoid was $38.44 \%$, as measured by the UV assay. The MTT assay (Table IV) demonstrated that both curcuminoid and HA/CNP treatments enhanced rat chondrocyte proliferation. Additionally, HA/CNP treatment exerted a stronger enhancing effect on chondrocyte proliferation compared with that of curcuminoid treatment. The $\mathrm{IC}_{50}$ of HA/CNP for the delivery of curcuminoid at $48 \mathrm{~h}$ was $8.93 \pm 1.14 \mu \mathrm{g} / \mathrm{ml}$. The in vitro bag filter method (Table V) showed that the cumulative release rate of curcuminoid after $1 \mathrm{~h}$ in PBS reached $78 \%$ and maintained that level over time. Under the same conditions, the release rate of HA/CNP for the delivery of curcuminoid increased from 40 to $74 \%$ over time. This result suggested that HA/CNP for the delivery of curcuminoid has a better sustained release function. TEM observation (Fig. 1) revealed that curcuminoid and HA/CNP deliveries were both ball-shaped and regular. The diameter of the curcuminoid particle was $42.46 \pm 3.23 \mathrm{~nm}$, while the diameter of HA/CNPs was $164.68 \pm 14.21 \mathrm{~nm}$. These three experiments demonstrated that HA/CNP for the delivery of curcuminoid was able to accelerate chondrocyte proliferation and has a sustained release.

Identification of rat chondrocytes. Primary culture of SD rat chondrocytes (Fig. 2A and B) demonstrated that rat chondrocytes were tightly packed and had an elongated spindle shape. Immunofluorescence assay revealed strong collagen II expression in rat chondrocytes (Fig. 2C and D). These results
Table IV. $\mathrm{IC}_{50}$ of curcuminoid and HA/CNP for delivery of curcuminoid to rat chondrocytes $(\mu \mathrm{g} / \mathrm{ml})$.

\begin{tabular}{lcr}
\hline Drug & $24 \mathrm{~h}$ & \multicolumn{1}{c}{$48 \mathrm{~h}$} \\
\hline Curcuminoid & $82.31 \pm 5.46$ & $46.75 \pm 5.73$ \\
HA/CNP for delivery of & $24.23 \pm 3.85$ & $8.93 \pm 1.14$ \\
curcuminoid & & \\
\hline
\end{tabular}

HA, hyaluronic acid; CNP, chitosan nanoparticle.
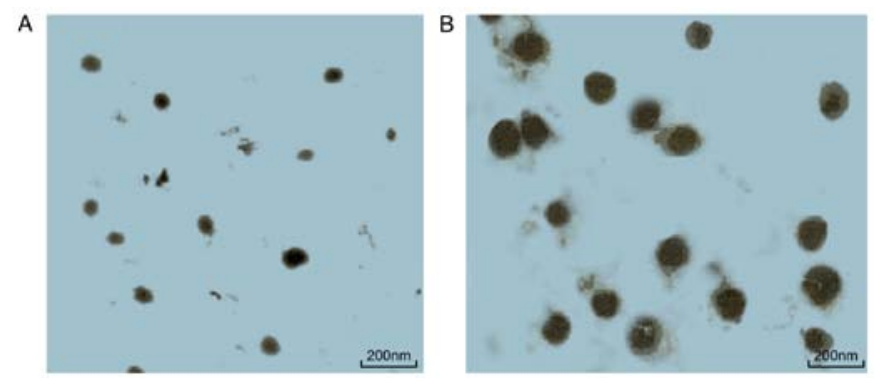

Figure 1. HA/CNP for the delivery of curcuminoid (B) was bigger compared with that for curcuminoid (A), while they were both spherical in shape, as observed by transmission electron microscopy (magnification, x50,000). HA, hyaluronic acid; CNP, chitosan nanoparticle.

demonstrated that the cells that were isolated and cultured were rat chondrocytes.

HA/CNP for the delivery of curcuminoid enhances proliferation but suppresses apoptosis and inflammatory response in chondrocytes. The biological functions of HA/CNP for the delivery of curcuminoid were detected by EdU assay, RT-qPCR assay, western blot analysis, P65 protein 
nuclear translocation detection and flow cytometry. The EdU assay (Fig. 3A) determined that the cell proliferation rate of the NC group was $13.62 \pm 1.22 \%$, the rate of the HA group was $20.33 \pm 1.83 \%$, the rate of the curcuminoid group was $19.14 \pm 2.12 \%$, while the rate of the HA/CNP for the delivery of curcuminoid group was $36.42 \pm 3.26 \%$. RT-qPCR (Fig. 3B) revealed that, compared with the NC group, the HA and curcuminoid groups exhibited increased collagen II mRNA levels but decreased MMP-1 and MMP-13 mRNA levels (all $\mathrm{P}<0.05$ ). Additionally, the HA/CNP group also exhibited significantly increased collagen II mRNA levels, but significantly decreased MMP-1 and MMP-13 mRNA levels (all $\mathrm{P}<0.01$ ). Western blot analysis (Fig. $3 \mathrm{C}$ and $\mathrm{D}$ ) demonstrated that, in comparison with the $\mathrm{NC}$ group, the protein levels of collagen II and I- $\mathrm{BB} \alpha$ were increased, but the protein levels of MMP-1 and MMP-13 and the extent of I- $\mathrm{kB} \alpha$ phosphorylation were decreased in the curcuminoid and HA groups. In addition, compared with the curcuminoid group, the protein levels of collagen II and I- $\kappa \mathrm{B} \alpha$ were elevated, while the protein levels of MMP-1 and MMP-13 and the extent of I- $\kappa \mathrm{B} \alpha$ phosphorylation were decreased in the HA/CNP group (all $\mathrm{P}<0.01$ ). P65 protein nuclear translocation detection (Fig. 3E and F) revealed that the curcuminoid and the HA groups had more P65 in the cytoplasm but less P65 in the cell nucleus compared with the NC group. Furthermore, the HA/CNP had more P65 in the cytoplasm but less P65 in the cell nucleus compared with the curcuminoid group (all $\mathrm{P}<0.01$ ). Flow cytometry analysis (Fig. 3G) showed that cell apoptosis rate was decreased in the HA and the curcuminoid groups $(\mathrm{P}<0.05)$ and significantly decreased in the HA/CNP group $(\mathrm{P}<0.01)$ when compared with the NC group, while the HA/CNP group had a lower cell apoptosis rate compared with the curcuminoid group $(\mathrm{P}<0.05)$. Taken together, our results suggest that HA/CNP for the delivery of curcuminoid enhanced proliferation but suppressed apoptosis and inflammatory response in chondrocytes.

Establishment of the OA model. SD rat behavioral observation showed that SD rats in the sham group walked normally, but $\mathrm{SD}$ rats in the OA group walked slowly with the bodies tilted to the right. Safranin O-fast green staining of knee joints (Fig. 4) showed hyaline cartilage and calcified cartilage in the knee joints (red) and subchondral bone (blue). The rats in the sham group had more calcified cartilage and matrix outside the chondrocytes, more obvious layers of chondrocytes, and a smoother surface, while rats in the OA group had no obvious boundary lines between the subchondral bone layer and calcified cartilage layer, with comb-like structures, lightly stained chondrocyte lacunae in the hyaline cartilage and scattered osteocytes in the subchondral bone zone. Collectively, these data demonstrated that the knee OA model in SD rats was successfully established.

HA/CNP alleviates knee joint lesions in rats with knee OA. Finally, the effect of HA/CNP delivery on knee joint lesions in rats with knee OA was investigated. RT-qPCR assay (Fig. 5A) revealed that, compared with the NC group, the mRNA levels of collagen II were elevated, while the mRNA levels of MMP-1 and MMP-13 were decreased in the HA group (all $\mathrm{P}<0.05$ ). Additionally, collagen II mRNA expression was 

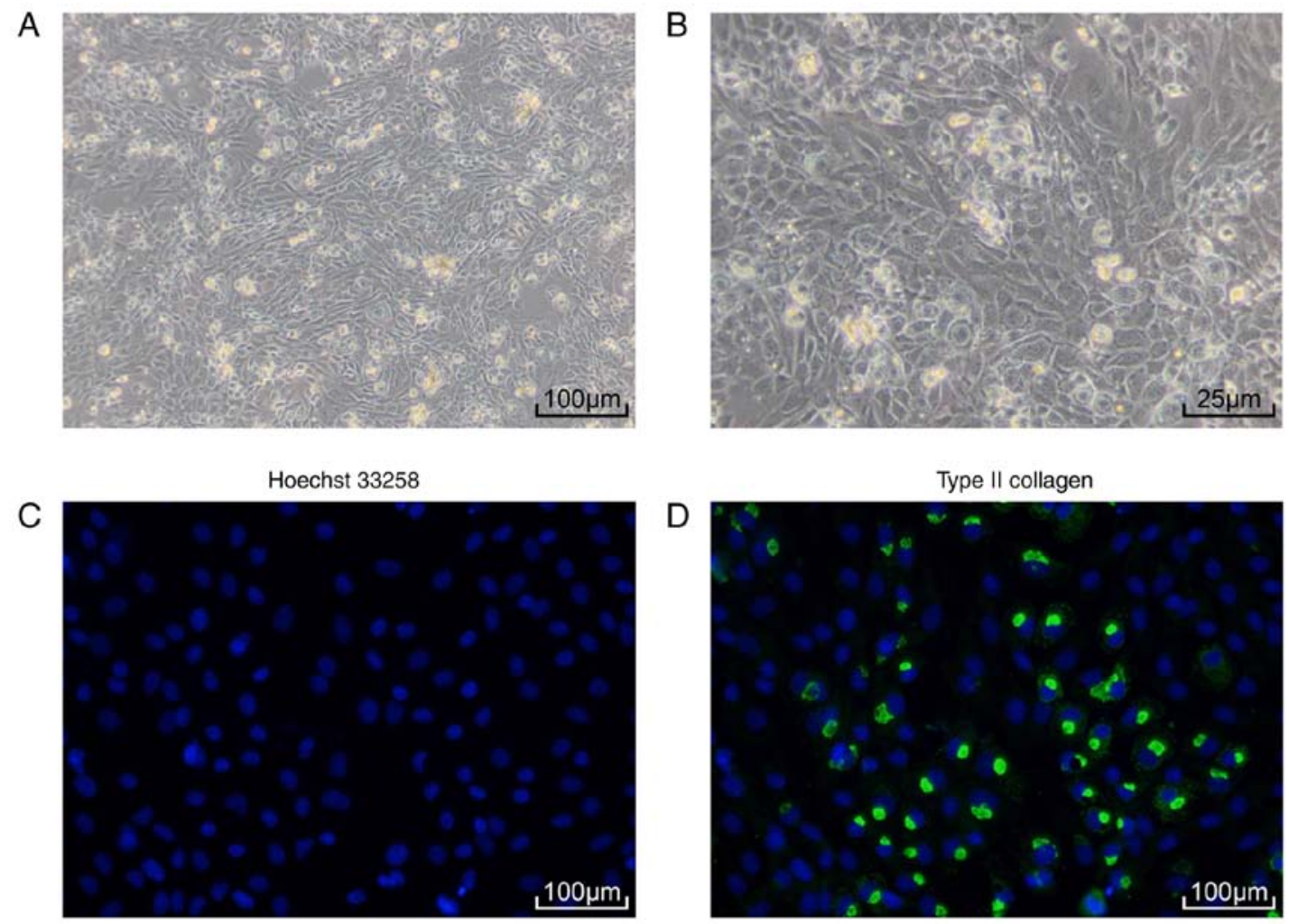

Figure 2. Identification of chondrocytes isolated from Sprague Dawley (SD) rats. (A) Morphology of third-generation SD rat chondrocytes observed under an inverted microscope (magnification, x100); (B) morphology of third-generation SD rat chondrocytes observed under an inverted microscope (magnification, x400); (C) chondrocytes exhibited increased expression of Hoechst 33258, as detected by immunofluorescence assay (magnification, x100); (D) chondrocytes exhibited increased expression of collagen II, as detected by immunofluoresence assay (magnification, x100).

significantly elevated and MMP-1 and MMP-13 expression was significantly decreased in the HA/CNP for the delivery of curcuminoid group (all $\mathrm{P}<0.01$ ). Western blot analysis (Fig. 5B and C) demonstrated that the HA and the HA/CNP for the delivery of curcuminoid groups had higher collagen II and $\mathrm{I}-\kappa \mathrm{B} \alpha$ protein levels, lower MMP-1 and MMP-13 protein levels, and decreased I- $\kappa \mathrm{B} \alpha$ phosphorylation compared with the NC group. It was also observed that SD rat knee joints in the NC group exhibited a rough surface and local erosion (Fig. 5D), while the knee joints in the HA group had a complete surface without gloss, and the knee joints in the HA/CNP group had a smooth and complete surface. H\&E staining (Fig. 5E) demonstrated that the articular cartilage surface layer was fiberized and cell lamination was completely disordered in the NC group, while the knee joint surface was partially incomplete and cells were partially disordered in the HA group, whereas the knee joint surface was smooth and the cells were regularly arranged in the HA/CNP group. Following toluidine blue staining (Fig. 5F), some of the layers were not dyed, and the chondrocytes were significantly decreased with disorderly layers in the NC group. In comparison, articular cartilage was lightly stained and the cells were somewhat disorderly but arranged in clearer layers in the HA group, while the knee joints were deeply stained blue, cartilages had a flat surface, the cells were orderly arranged and there were distinct layers in the HA/CNP group. Following Safranin O-fast green staining (Fig. 5G), some of the layers were not dyed and the chondrocytes were significantly decreased with disordered layers in the NC group, while articular cartilage was lightly stained and the cells were somewhat disordered, but in clearer layers in the HA group. However, the knee joints were deeply stained with red, the cartilages had a flat surface, the cells were orderly arranged and there were distinct layers in the HA/CNP group. The Outerbridge classification and Mankin pathological score of knee chondrocytes are shown in Table VI. Taken together, these results indicate that HA/CNP alleviates knee joint lesions in rats with knee OA.

\section{Discussion}

As a common treatment for knee OA, HA has been recently adopted as a ligand to contribute to drug delivery (17). Therefore, HA was selected as the ligand for curcuminoid, an effective drug for knee OA therapy. In addition, CNP may be used to improve curcuminoid stability and solubility (10). As a result, it was decided to prepare a new combination of drugs, HA/CNP, for curcuminoid delivery and explore its therapeutic effect in this study. The results demonstrated that HA/CNP for the delivery of curcuminoid inhibited inflammation and

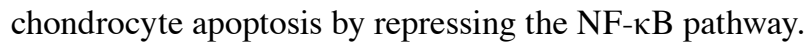

The major finding of the present study was that HA/CNP decreased chondrocyte apoptosis by inhibiting the $\mathrm{NF}-\kappa \mathrm{B}$ pathway. The NF- $\mathrm{NB}$ pathway, as one of the most important catabolic pathways, participates in the pathogenesis of OA and critically regulates inflammatory mediators that are associated with OA (18). It was previously demonstrated that the combination of HA and curcuminoid can act synergistically to reduce scar formation (19). Ji et al demonstrated 

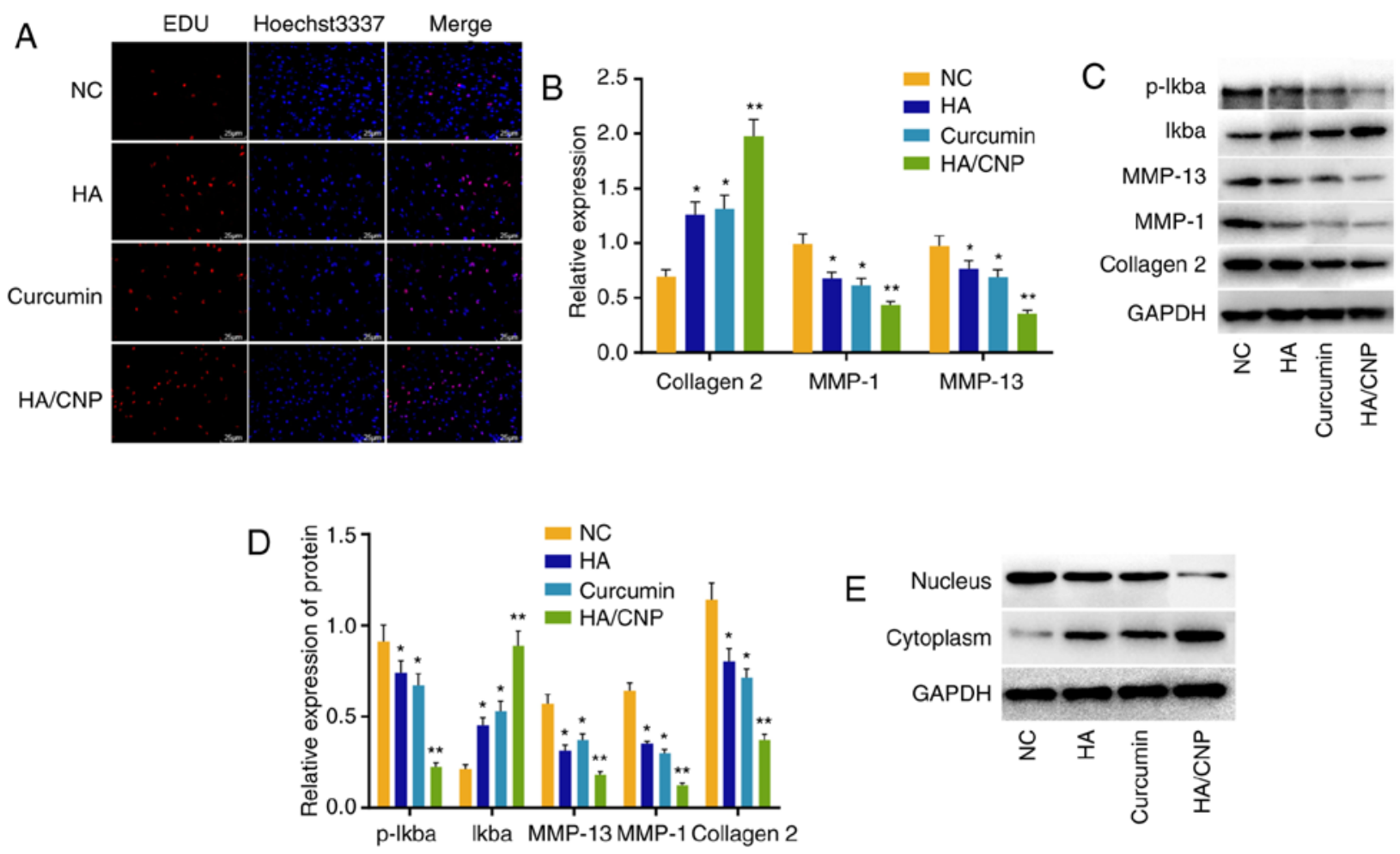

\section{F}
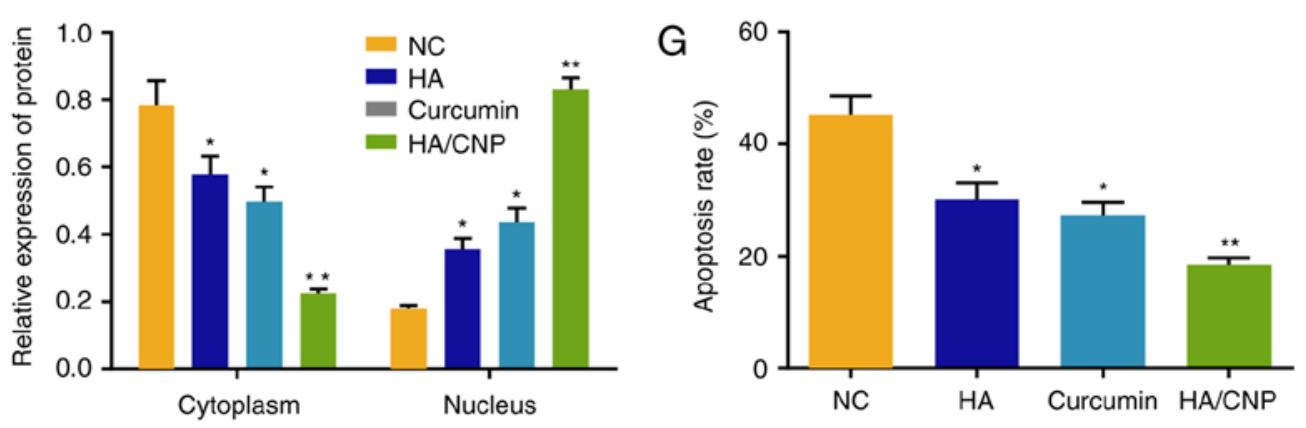

Figure 3. HA/CNP for the delivery of curcuminoid enhances cell proliferation but suppresses apoptosis and inflammatory responses in chondrocytes. (A) EdU assay revealed that $\mathrm{HA} / \mathrm{CNP}$ for the delivery of curcuminoid enhanced chondrocyte proliferation; (B) RT-qPCR assay demonstrated that HA and curcuminoid increased collagen II mRNA levels but decreased MMP-1 and MMP-13 mRNA levels, and that HA/CNP for the delivery of curcuminoid exerted stronger effects compared with individual treatments. (C and D) Western blot analysis showed that HA and curcuminoid treatment elevated the protein levels of collagen II and I- $\mathrm{\kappa} \mathrm{B} \alpha$, but reduced the protein levels of MMP-1 and MMP-13, while I- $\mathrm{\kappa B} \alpha$ phosphorylation was decreased. HA/CNP treatment significantly enhanced the response compared with the two individual treatments. (E and F) Western blot analysis revealed that HA/CNP for the delivery of curcuminoid promoted P65 translocation from the cell nucleus to the cytoplasm. (G) Flow cytometry demonstrated that HA/CNP for the delivery of curcuminoid inhibited chondrocyte apoptosis; ${ }^{*} \mathrm{P}<0.05$ and ${ }^{* *} \mathrm{P}<0.01$ compared with the $\mathrm{NC}$ group. Measurement data, including mRNA and protein levels, cell proliferation rate and cell apoptosis rate, were all expressed by the mean value \pm standard deviation. Statistical analysis was performed using one-way ANOVA; $n=3$ replicates per experiment for 3 experiments. RT-qPCR, reverse transcription quantitative polymerase chain reaction; MMP, matrix metalloproteinase; NC, negative control; ANOVA, analysis of variance; $\mathrm{HA}$, hyaluronic acid; CNP, chitosan nanoparticle.
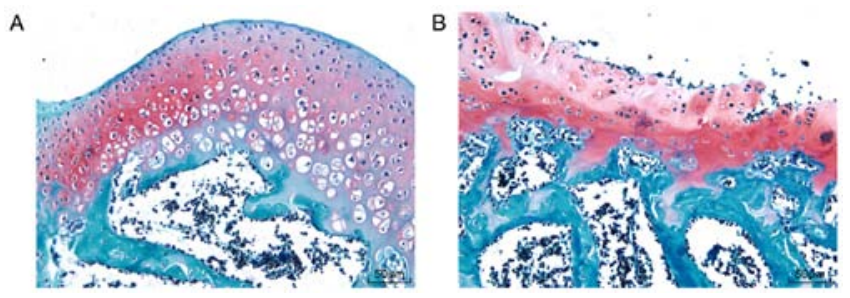

Figure 4. OA models of 5 Sprague Dawley rats were successfully established and verified by safranin O-fast green staining. (A) Cartilaginous tissue in the sham group displayed more calcified cartilage and matrix outside the chondrocytes, distinct chondrocyte layers and smooth surface. (B) Cartilaginous tissue in the OA group exhibited no obvious boundary lines between the subchondral bone layer and the calcified cartilage layer, with comb-like structures, lightly stained chondrocyte lacunae in hyaline cartilage, and scattered osteocytes in the subchondral bone zone. OA, osteoarthritis. that isoliquiritigenin can protect chondrocytes and prevent inflammation by decreasing the activation of the $N F-\kappa B$ pathway (4). Zhuang et al demonstrated that kaempferol may be a good therapeutic strategy for OA, since it can inhibit $\mathrm{NF}-\kappa \mathrm{B}$ and decrease IL-1 $\beta$-stimulated inflammation in chondrocytes of rats with OA (20). Importantly, it is obvious that the inhibition of $\mathrm{NF}-\kappa \mathrm{B}$ pathway can be a point to explore the OA therapy. In the present study, HA/CNP for the delivery of curcuminoid was found to promote the expression of $\mathrm{I} \kappa \mathrm{B}$. $\mathrm{I} \kappa \mathrm{B}$ is an inhibitor of the $\mathrm{NF}-\kappa \mathrm{B}$ pathway (21). Therefore, $\mathrm{HA} / \mathrm{CNP}$ can be used to inhibit the NF- $\mathrm{B}$ pathway by increasing I $\mathrm{B}$. Moreover, Li et al have proved that inhibiting phosphorylated P65 translocation to the nucleus can promote the effect of baicalein against chondrocyte inflammation 

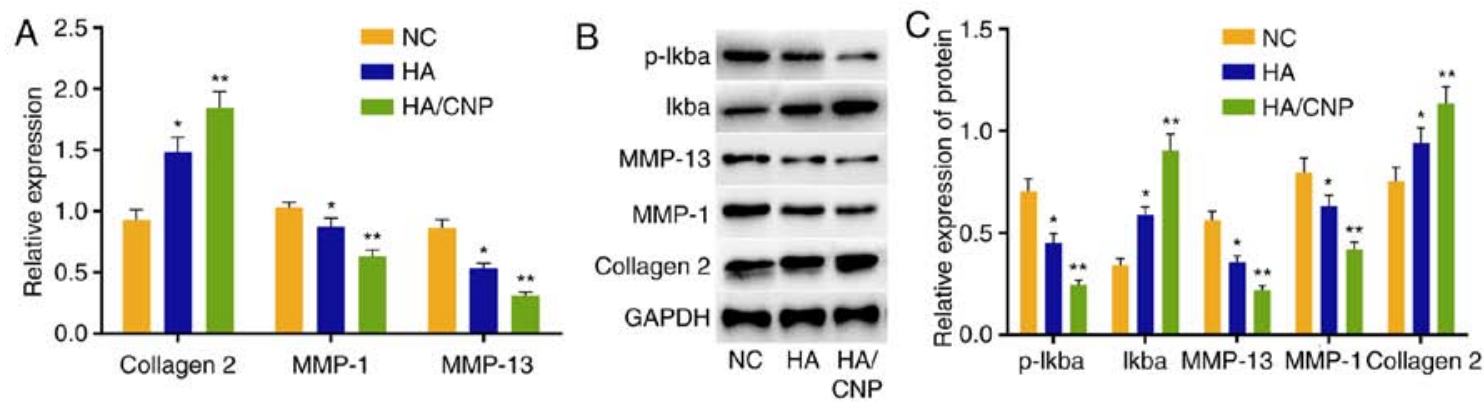

D
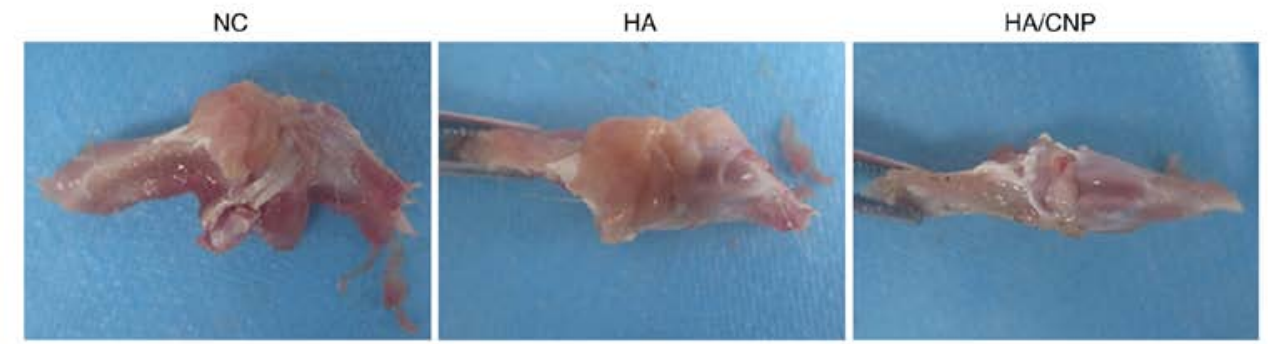

E
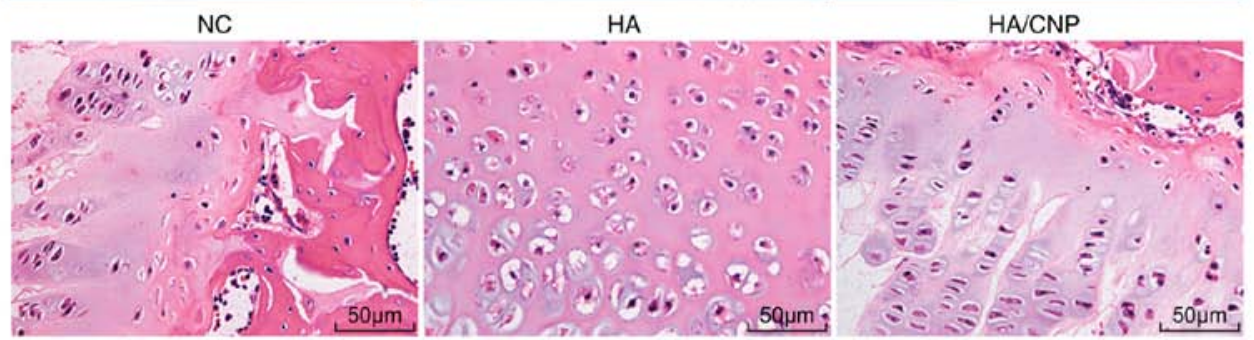

$\mathrm{F}$
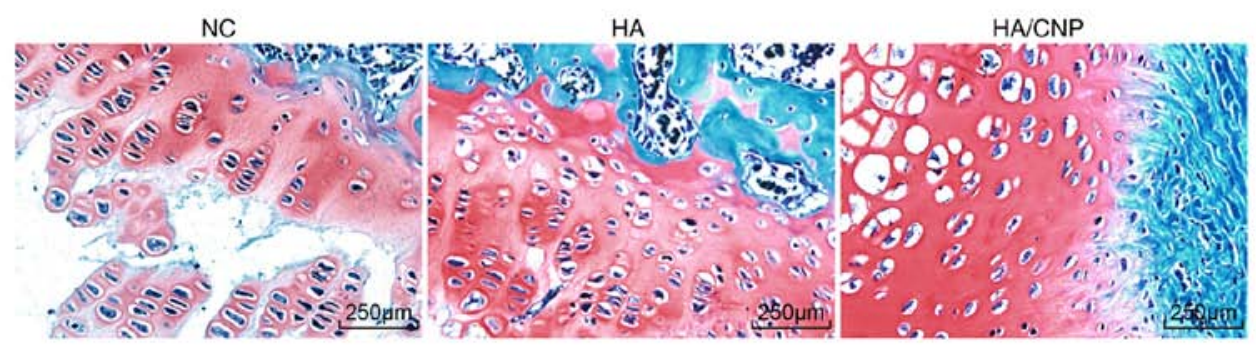

G
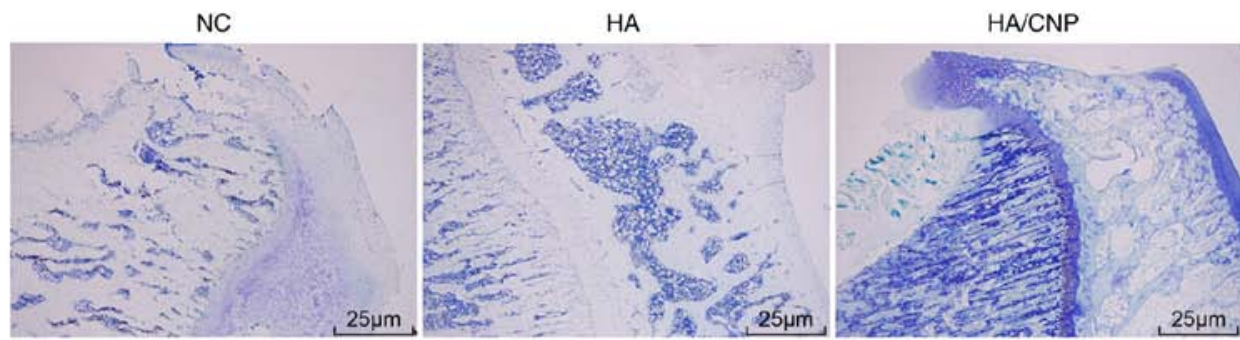

Figure 5. HA/CNP for delivery of curcuminoid confers protection against knee joint lesions in rats with knee OA. (A) RT-qPCR assay showed that collagen II mRNA level was significantly increased and MMP-1 and MMP-13 mRNA levels in chondrocytes were significantly decreased after treatment with HA/CNP for delivery of curcuminoid. (B and C) Western blot analysis demonstrated that collagen II and I- $\mathrm{kB} \alpha$ protein levels were significantly increased and MMP-1 and MMP-13 protein levels as well as the extent of I- $\mathrm{k} B \alpha$ phosphorylation were decreased after treatment with $\mathrm{HA} / \mathrm{CNP}$ for delivery of curcuminoid. (D) Under a microscope, HA/CNP for delivery of curcuminoid improved articular surface injury in OA rats. (E) H\&E staining revealed that the knee joint surface was smooth and the cells were regularly arranged in OA rats treated with HA/CNP for delivery of curcuminoid (x400). (F) Toluidine blue staining revealed that knee joints are deeply stained with blue, the cartilages had a flat surface, the cells were orderly arranged and there were distinct layers in OA rats treated with HA/CNP for delivery of curcuminoid (x40). (G) Safranin O-fast green staining demonstrated that the knee joints were deeply stained with red, the cartilages had a flat surface, the cells were orderly arranged and there were distinct layers in OA rats treated with HA/CNP for delivery of curcuminoid (x400); ${ }^{*} \mathrm{P}<0.05$ and ${ }^{* *} \mathrm{P}<0.01$ compared with the NC group. mRNA and protein value, as measurement data, were all expressed by the mean value \pm standard deviation, the result of which were verified by one-way ANOVA; $n=3$; the experiments were repeated 3 times. H\&E, hematoxylin and eosin; HA, hyaluronic acid; RT-qPCR, reverse transcription quantitative polymerase chain reaction; MMP, matrix metalloproteinase; NC, negative control; ANOVA, analysis of variance; OA, osteoarthritis; CNP, chitosan nanoparticle.

and apoptosis (22). Therefore, inhibiting P65 translocation to the nucleus may be a target for OA therapy. Interestingly, this study has demonstrated that HA/CNP for the delivery of curcuminoid was able to relocate P65 from the cell nucleolus to the cytoplasm, which provides evidence that HA/CNP for the delivery of curcuminoid may be an optimal drug for OA 
Table VI. Outerbridge classification and Mankin pathological score of Sprague Dawley rat knee-joint chondrocytes.

\begin{tabular}{|c|c|c|c|}
\hline Drug treatment & Period (week) & Outerbridge classification & Mankin pathological score \\
\hline \multirow[t]{4}{*}{$\mathrm{NC}$} & 1 & II & 7 \\
\hline & 2 & II & 7 \\
\hline & 3 & II & 7 \\
\hline & 4 & II & 7 \\
\hline \multirow[t]{4}{*}{ HA } & 1 & II & 7 \\
\hline & 2 & II & 6 \\
\hline & 3 & I & 4 \\
\hline & 4 & I & 3 \\
\hline \multirow[t]{4}{*}{ HA/CNP for delivery of curcuminoid } & 1 & I & 3 \\
\hline & 2 & I & 2 \\
\hline & 3 & 0 & 1 \\
\hline & 4 & 0 & 0 \\
\hline
\end{tabular}

NC, negative control; HA, hyaluronic acid; CNP, chitosan nanoparticle.

therapy. However, Hwang et al reported that the inhibition of chondrocyte apoptosis, which is the main pathogenetic mechanism, can be targeted in OA therapy by modulating cartilage degeneration (23). Our study also demonstrated that HA/CNP for the delivery of curcuminoid can decrease chondrocyte apoptosis and knee joint lesions in knee OA. NF- $\mathrm{BB}$ regulates inflammatory responses and mediates related chondrocyte inflammatory responses, which may cause cartilage degradation (24). Our findings revealed that HA/CNP for the delivery of curcuminoid suppresses chondrocyte apoptosis by inactivating the $\mathrm{NF}-\kappa \mathrm{B}$ pathway.

Another important finding of the present study was that HA/CNP for the delivery of curcuminoid inhibited the expression of inflammation-related genes, such as MMP-1 and MMP-13, but promoted collagen II expression. Collagen II is the major component of articular cartilage. Sufficient collagen II is crucial for cartilage biomechanical properties (25), while previous findings determined that MMP-13 is the primary protein involved in cartilage degradation in the course of OA by cleaving collagen II (26). MMP-1 degrades type I, II, III, VII, X, IX and other types of collagen and aggrecan, while MMP-13 mainly cleaves collagen type II, IV and IX, osteonectin and perlecan in the cartilage (27). When stimulated by catabolism and anabolism, HA suppresses MMP-1 and MMP-13 (28). Curcuminoid may inhibit downstream MMP-1 and MMP-13 gene upregulation by oncostatin $M$ in chondrocytes when upstream STAT1 phosphorylation is inhibited (29). Furthermore, our results demonstrated that HA/CNP for the delivery of curcuminoid inhibited the expression of MMP-1 and MMP-13, and promoted collagen II expression.

In conclusion, the present study suggests that intra-articular HA/CNP for the delivery of curcuminoid can decrease chondrocyte apoptosis in rats with knee OA through repression of the NF- $\kappa \mathrm{B}$ pathway. These findings may lead to novel OA therapies in the future. However, it should be noted that this study has examined only SD rat models; therefore, it is not clear at present if there are unwanted side effects in practical clinic applications. Due to time and funding constraints, we did not study the effect of inhibition of the $N F-\kappa B$ pathway on arthritis models. Further studies may focus on the effects of inhibition of the NF- $\mathrm{B}$ pathway and other pathways on arthritis models. It was demonstrated in rats that HA/CNP for the delivery of curcuminoid may be a potential therapy for OA, which may represent a creative suggestion to doctors. Furthermore, we will continue to explore materials that can further improve the solubility and stability of HA and curcuminoid.

\section{Acknowledgements}

The authors are grateful to all the staff who contributed to this study and the construction plan of characteristic medical specialties and community projects of Baoshan District.

\section{Funding}

The present study was supported by Science and Technology Development Fund Project of Baoshan District (17-E-1); Scientific Research Project of the Shanghai Municipal Health and Planning Committee (20174Y0240).

\section{Availability of data and materials}

The data generated and analyzed in the present study are available from the corresponding author upon reasonable request.

\section{Authors' contributions}

JW conceived and together with XW and YC designed the study. TH and DXS were involved in data collection. XW and HRT performed the statistical analysis and figure preparation. YC and TH drafted the manuscript. JW, XW, TH and DXS performed critical revision of the article. All authors read and approved the final version of the manuscript. 


\section{Ethics approval and consent to participate}

The breeding and sacrifice of the experimental animals, as well as tissue retrieval, were all for medical research and performed in strict accordance with the recommendations in the Guide for the Care and Use of Laboratory Animals of the National Institutes of Health. The procedures were approved by the Animal Committee of Shanghai Ninth People's Hospital, Shanghai Jiao Tong University School of Medicine.

\section{Patient consent for publication}

Not applicable.

\section{Competing interests}

The authors declare that they have no competing interests to disclose.

\section{References}

1. Wu Q, Sun X and Du L: Association of fibulin-3 concentrations with the presence and severity of knee osteoarthritis: A cross-sectional study. Knee 24: 1369-1373, 2017.

2. Ogunbode AM, Adebusoye LA, Olowookere OO and Alonge TO: Physical functionality and self-rated health status of adult patients with knee osteoarthritis presenting in a primary care clinic. Ethiop J Health Sci 24: 319-328, 2014.

3. Runhaar J, van Middelkoop M, Reijman M, Willemsen S, Oei EH, Vroegindeweij D, van Osch G, Koes B and Bierma-Zeinstra SM: Prevention of knee osteoarthritis in overweight females: The first preventive randomized controlled trial in osteoarthritis. Am J Med 128: 888-895.e4, 2015.

4. Ji B, Guo W, Ma H, Xu B, Mu W, Zhang Z, Amat A and Cao L: Isoliquiritigenin suppresses IL-1 $\beta$ induced apoptosis and inflammation in chondrocyte-like ATDC5 cells by inhibiting NF- $\kappa \mathrm{B}$ and exerts chondroprotective effects on a mouse model of anterior cruciate ligament transection. Int J Mol Med 40: 1709-1718, 2017.

5. Ricci M, Micheloni GM, Berti M, Perusi F, Sambugaro E, Vecchini E and Magnan B: Clinical comparison of oral administration and viscosupplementation of hyaluronic acid (HA) in early knee osteoarthritis. Musculoskelet Surg 101: 45-49, 2017.

6. Zhang Z, Leong DJ, Xu L, He Z, Wang A, Navati M, Kim SJ, Hirsh DM, Hardin JA, Cobelli NJ, et al: Curcumin slows osteoarthritis progression and relieves osteoarthritis-associated pain symptoms in a post-traumatic osteoarthritis mouse model. Arthritis Res Ther 18: 128, 2016.

7. Salwowska NM, Bebenek KA, Żąło DA and WcisłoDziadecka DL: Physiochemical properties and application of hyaluronic acid: A systematic review. J Cosmet Dermatol 15: 520-526, 2016

8. Concoff A, Sancheti P, Niazi F, Shaw P and Rosen J: The efficacy of multiple versus single hyaluronic acid injections: A systematic review and meta-analysis. BMC Musculoskelet Disord 18: 542, 2017.

9. Li X, Feng K, Li J, Yu D, Fan Q, Tang T, Yao X and Wang X: Curcumin inhibits apoptosis of chondrocytes through activation ERK1/2 signaling pathways induced autophagy. Nutrients 9: E414, 2017.

10. Wang F, Yang Y, Ju X, Udenigwe CC and He R: Polyelectrolyte complex nanoparticles from chitosan and acylated rapeseed cruciferin protein for curcumin delivery. J Agric Food Chem 66: 2685-2693, 2018.

11. $\mathrm{Yu} \mathrm{H}$, Nguyen $\mathrm{MH}$ and Hadinoto K: Effects of chitosan molecular weight on the physical and dissolution characteristics of amorphous curcumin-chitosan nanoparticle complex. Drug Dev Ind Pharm 44: 82-88, 2018.

12. Yasuda T: Type II collagen peptide stimulates Akt leading to nuclear factor-kappaB activation: Its inhibition by hyaluronan. Biomed Res 35: 193-199, 2014.

13. Yang $\mathrm{Q}, \mathrm{Wu} \mathrm{S}, \mathrm{Mao} X$, Wang $\mathrm{W}$ and Tai $\mathrm{H}$ : Inhibition effect of curcumin on TNF- $\alpha$ and MMP-13 expression induced by advanced glycation end products in chondrocytes. Pharmacology 91: 77-85, 2013.
14. Zhou L, Hu Y, Li C, Yan Y, Ao L, Yu B, Fang W, Liu J and Li Y: Levo-corydalmine alleviates vincristine-induced neuropathic pain in mice by inhibiting an NF-kappa B-dependent CXCL1/CXCR2 signaling pathway. Neuropharmacology 135: 34-47, 2018.

15. Xu H, Bouta EM, Wood RW, Schwarz EM, Wang Y and Xing L: Utilization of longitudinal ultrasound to quantify joint soft-tissue changes in a mouse model of posttraumatic osteoarthritis. Bone Res 5: 17012, 2017.

16. Xu J, Lu MX, Cui YD and Du YZ: Selection and evaluation of reference genes for expression analysis using qRT-PCR in Chilo suppressalis (Lepidoptera: Pyralidae). J Econ Entomol 110: 683-691, 2017.

17. Xu Y, Asghar S, Yang L, Chen Z, Li H, Shi W, Li Y, Shi Q, Ping Q and Xiao Y: Nanoparticles based on chitosan hydrochloride/hyaluronic acid/PEG containing curcumin: In vitro evaluation and pharmacokinetics in rats. Int J Biol Macromol 102: 1083-1091, 2017.

18. Chen C, Zhang C, Cai L, Xie H, Hu W, Wang T, Lu D and Chen H: Baicalin suppresses IL-1 $\beta$-induced expression of inflammatory cytokines via blocking NF- $\mathrm{KB}$ in human osteoarthritis chondrocytes and shows protective effect in mice osteoarthritis models. Int Immunopharmacol 52: 218-226, 2017.

19. Sharma M, Sahu K, Singh SP and Jain B: Wound healing activity of curcumin conjugated to hyaluronic acid: In vitro and in vivo evaluation. Artif Cells Nanomed Biotechnol 46: 1009-1017, 2018.

20. Zhuang Z, Ye G and Huang B: Kaempferol alleviates the interleukin-1 $\beta$-induced inflammation in rat osteoarthritis chondrocytes via suppression of NF- $\kappa$ B. Med Sci Monit 23: 3925-3931, 2017.

21. De Palma A, Cheleschi S, Pascarelli NA, Giannotti S, Galeazzi M and Fioravanti A: Hydrostatic pressure as epigenetic modulator in chondrocyte cultures: A study on miRNA-155, miRNA-181a and miRNA-223 expression levels. J Biomech 66: 165-169, 2018.

22. Li Y, Wang J, Song X, Bai H, Ma T, Zhang Z, Li X, Jiang R, Wang G, Fan X, et al: Effects of baicalein on IL-1 $\beta$-induced inflammation and apoptosis in rat articular chondrocytes. Oncotarget 8: 90781-90795, 2017.

23. Hwang HS and Kim HA: Chondrocyte apoptosis in the pathogenesis of osteoarthritis. Int J Mol Sci 16: 26035-26054, 2015.

24. Lee HG and Yang JH: PCB126 induces apoptosis of chondrocytes via ROS-dependent pathways. Osteoarthritis Cartilage 20: 1179-1185, 2012.

25. Ito $\mathrm{K}$ and Shinomura T: Development and application of a new Silent reporter system to quantitate the activity of enhancer elements in the type II collagen gene. Gene 585: 13-21, 2016.

26. Motomura H, Seki S, Shiozawa S, Aikawa Y, Nogami M and Kimura T: A selective c-Fos/AP-1 inhibitor prevents cartilage destruction and subsequent osteophyte formation. Biochem Biophys Res Commun 497: 756-761, 2018.

27. Stancker TG, Vieira SS, Serra AJ, do Nascimento Lima R, Dos Santos Feliciano R, Silva JA Jr, Dos Santos SA, Dos Santos Vieira MA, Simões MCB, Leal-Junior EC, et al: Can photobiomodulation associated with implantation of mesenchymal adipose-derived stem cells attenuate the expression of MMPs and decrease degradation of type II collagen in an experimental model of osteoarthritis? Lasers Med Sci 33: 1073-1084, 2018.

28. Pohlig F, Guell F, Lenze U, Lenze FW, Mühlhofer HM, SchauweckerJ, ToepferA,Mayer-KuckukP, vonEisenhart-RotheR, Burgkart R and Salzmann GM: Hyaluronic acid suppresses the expression of metalloproteinases in osteoarthritic cartilage stimulated simultaneously by interleukin $1 \beta$ and mechanical load. PLoS One 11: e0150020, 2016.

29. Li WQ, Dehnade F and Zafarullah M: Oncostatin M-induced matrix metalloproteinase and tissue inhibitor of metalloproteinase-3 genes expression in chondrocytes requires Janus kinase/STAT signaling pathway. J Immunol 166: 3491-3498, 2001.

This work is licensed under a Creative Commons Attribution-NonCommercial-NoDerivatives 4.0 International (CC BY-NC-ND 4.0) License. 